\title{
Indicadores de boas práticas durante a assistência à mulher no trabalho de parto $\mathrm{e}$
}

\section{nascimento}

\author{
Indicators of good practices during assistance to women in labor and birth \\ Indicadores de buenas prácticas durante la atención a la mujer en el trabajo de parto y nacimiento
}

Recebido: 03/01/2022 | Revisado: 09/01/2022 | Aceito: 15/01/2022 | Publicado: 17/01/2022

Nara Beatriz Silveira

ORCID: https://orcid.org/0000-0001-9723-7845 Universidade Federal do Rio Grande, Brasil

E-mail: nara.beatriz@hotmail.com

Vanessa Machado da Silva

ORCID: https://orcid.org/0000-0002-4319-4509 Universidade Federal do Rio Grande, Brasil

E-mail: va.nessamachado@outlook.com

Francisca Alice Cunha Rodrigues

ORCID: https://orcid.org/0000-0002-2765-929X

Universidade Federal do Rio Grande, Brasil

E-mail:alice.cunha1@hotmail.com

Sibele Schaun

ORCID: https://orcid.org/0000-0001-8985-6144

Universidade Federal do Rio Grande, Brasil

E-mail: sibele_schaun@yahoo.com

Rosemary Silva da Silveira

ORCID: https://orcid.org/0000-0003-0671-0022

Universidade Federal do Rio Grande, Brasil

E-mail: rosemaryssilveira@gmail.com

\begin{abstract}
Resumo
Objetivo: avaliar os indicadores de boas práticas durante a assistência à mulher em trabalho de parto e nascimento. Método: estudo quantitativo, documental, descritivo, com delineamento transversal. Na coleta de dados, utilizou-se uma pesquisa documental, no período de abril a setembro de 2019, avaliando os indicadores de boas práticas prestadas as gestantes em trabalho de parto. A amostra constitui-se de 100\% dos partos ocorridos no período estudado, independentemente de ser parto natural ou cesárea. Para a análise dos dados, realizou-se a estatística descritiva, com frequência absoluta e relativa. Resultados: constatou-se que apesar das boas práticas, prevalecem altos índices de intervenções não recomendadas pelo Ministério da Saúde. Pode-se constatar que, apesar da recente inclusão das enfermeiras obstétricas neste serviço, o índice de boas práticas desenvolvidas por essas profissionais foi de $100 \%$. Conclusão: percebe-se que existe um grande desafio na busca da transformação da assistência às mulheres e aos recémnascidos pautada nas boas práticas preconizadas pelo Ministério da Saúde.
\end{abstract}

Palavras-chave: Humanização; Parto normal; Enfermagem obstétrica; Indicadores de qualidade; Qualidade da assistência à saúde.

\begin{abstract}
Objective: to evaluate the indicators of good practices during the assistance to women in labor and birth. Method: quantitative, documentary, descriptive study with a cross-sectional design. Using documentary research in data collection, evaluating the indicators of good practices provided to pregnant women in labor from April to September 2019. The sample consists of $100 \%$ of births occurring during the study period regardless of it was a natural birth or a cesarean section. For data, analysis descriptive statistics are performed with absolute and relative frequency. Results: establishes that despite good practices, high amounts of interventions not recommended by the Ministry of Health prevail. Despite the recent inclusion of obstetric nurses in this service, it is perceptible the rate of good practices developed by these professionals was $100 \%$. Conclusion: it has been noticed that there is a great challenge in the search for the transformation of assistance to women and newborns based on the good practices recommended by the Ministry of Health.
\end{abstract}

Keywords: Humanization; Normal birth; Obstetric nursing; Quality Indicators; Quality of health care.

\section{Resumen}

Objetivo: evaluar los indicadores de buenas prácticas em la atención a la mujer en trabajo de parto y nacimiento. Método: estudio cuantitativo, documental, descriptivo con diseño transversal. En la recolección de datos, se ha utilizado una 
investigación documental, desde abril hasta septiembre de 2019, evaluando los indicadores de buenas prácticas brindadas a las gestantes en trabajo de parto. La muestra está compuesta por el $100 \%$ de los nacimientos que tuvieron lugar durante el período del estudio, independientemente si fue un parto natural o una cesárea. Para el análisis de los datos, se han realizado estadísticas descriptivas, con frecuencia absoluta y relativa. Resultados: se ha constatado que, a pesar de las buenas prácticas, prevalecen altos índices de intervenciones no recomendadas por el Ministerio de Salud, se puede observar que, a pesar de la reciente incorporación de enfermeras obstétricas en este servicio, el índice de buenas prácticas desarrollado por estos profesionales fue del $100 \%$. Conclusión: se ha percibido que hay un gran desafío en la búsqueda de la transformación de la asistencia a la mujer y al recién nacido basado en las buenas prácticas recomendadas por el Ministerio de Salud.

Palabras clave: Humanización; Parto normal; Enfermería obstétrica; Indicadores de calidad; Calidad de la atención de la salud.

\section{Introdução}

O Brasil tem realizado investimentos para promover as práticas de assistência à mulher no ciclo gravídico puerperal, de modo a evitar a realização de intervenções desnecessárias, o isolamento das pacientes de seus familiares, a falta de autonomia e decisão das mulheres sobre seu corpo. O Ministério da Saúde (MS) tem demonstrado que os índices de mortalidade materna no ciclo puerperal no Brasil, ainda são preocupantes (Brasil, 2020). Aliado a isso, na prática, não se alcança a totalidade das boas práticas assistenciais, tendo em vista que estas ainda são pautadas na medicalização, verticalização de programas e fragmentação de ações (Oliveira \& Penna, 2017).

Partindo do pressuposto que a integralidade da assistência é um dos princípios norteadores das práticas e serviços que compõe o Sistema Único de Saúde (SUS), e seguindo a lógica do cuidado integral, que considera as dimensões biológicas, psicológicas e sociais do indivíduo, ao longo das últimas décadas o MS tem proposto diretrizes, normas e protocolos, a fim de garantir a melhora no modelo de atendimento obstétrico com estimulação de práticas menos intervencionistas. Acredita-se que os altos índices de mortalidade no ciclo gravídico puerperal, estão diretamente relacionados a ausência de boas práticas, através de intervenções desnecessárias ainda praticadas pelos profissionais de saúde nas instituições hospitalares (Brasil, 2020).

A partir da premissa, que para melhorar os indicadores de atendimento no ciclo gravídico puerperal, seriam necessárias melhorias no atendimento a essas mulheres, o Brasil inicia a qualificação desse modelo intervencionista, buscando o uso apropriado das tecnologias, bem como, capacitando os profissionais de saúde. Dessa forma, na busca por essa qualificação na assistência no ciclo gravídico puerperal, o MS em parceria com diferentes órgãos governamentais, lança o projeto "Rede Cegonha" buscando um enfrentamento da violência obstétrica, dos altos índices de mortalidade materna, e da baixa qualidade do atendimento mostrada nos índices do MS nas redes públicas de atenção ao parto do País (Brasil, 2011; 2015).

Do mesmo modo, preconiza algumas diretrizes para guiar as ações dos profissionais, enfatizando as boas práticas que devem ser consideradas eficazes e estimuladas; chama a atenção para as práticas comprovadamente ineficazes e prejudiciais que devem ser evitadas, para as práticas que possuem poucas evidências científicas e que devem ser utilizadas com cautela e, ainda, para as práticas que frequentemente são utilizadas inapropriadamente (Brasil, 2017).

Dentre as boas práticas que devem ser estimuladas estão a posição não litotômica, a presença do acompanhante, o contato pele a pele ao nascer entre a mãe e o bebê e a amamentação na primeira hora de vida. Deve-se evitar a Manobra de Kristeller e usar com cautela a administração de ocitocina e episiotomia (Brasil, 2017). Tendo em vista que a instituição pesquisada está em processo de inserção das boas práticas torna-se imprescindível que os indicadores sejam analisados e usados no sentido de auxiliar na melhoria da assistência, na busca por um atendimento humanizado e de qualidade.

Sendo assim, justifica-se a realização deste estudo diante a necessidade de refletir sobre intervenções não preconizadas pelo MS na assistência à mulher no trabalho de parto e pós-parto imediato que ainda fazem parte da rotina dos profissionais de saúde, tendo o mesmo, como objetivo, analisar os indicadores de boas práticas de assistência à mulher no trabalho de parto e pós-parto imediato em um hospital de ensino no estado do Rio Grande do Sul. 


\section{Metodologia}

Trata-se de um estudo quantitativo, documental, do tipo descritivo, com delineamento transversal.

Estatísticas descritivas fornecem um resumo conciso dos dados, podendo ser resumidos de forma numérica ou gráfica, empregando rígidos recursos e técnicas estatísticas para classificá-los e analisá-los, tais como a porcentagem, a média, o desvio padrão, o coeficiente de correlação e as regressões, entre outros (Vogt et al., 2017).

A pesquisa quantitativa nos permite mensurar, hábitos, atitudes, ações, opiniões em determinado universo, através de uma amostra que a represente estatisticamente. Esse tipo de pesquisa, tem como características obedecer a um plano preestabelecido coma finalidade de enumerar eventos. Utiliza amostra, uma população especifica e instrumento para coleta de dados (Vogt et al., 2017).

Em razão de sua maior precisão e confiabilidade, os estudos quantitativos são mais indicados para o planejamento de ações coletivas, pois seus resultados são passíveis de generalização, principalmente quando as amostras pesquisadas representam, com fidelidade, a população de onde foram retiradas. Assim, o resultado da pesquisa quantitativa baseia-se na interpretação objetiva da realidade (Vogt et al., 2017).

A amostra foi constituída a partir dos registros online no sistema de registro local da instituição pesquisada, que constam os indicadores de boas práticas prestadas as gestantes que tiveram seus filhos na instituição, durante o período de abril a setembro de 2019. No tamanho amostral foi utilizado $100 \%$ dos partos ocorridos no período estudado, independentemente de ser parto natural ou cesárea.

A coleta de dados incluiu os seguintes itens: presença de acompanhante, assistência por enfermeira obstétrica ou médico obstetra, posição da mulher no momento do parto, realização de episiotomia e da Manobra de Kristeller, dieta no trabalho de parto, uso de punção venosa, uso de ocitocina, uso de métodos não farmacológicos para dor, tempo de clampeamento do cordão umbilical, realização de contato pele a pele entre mãe e recém-nascido e amamentação na primeira hora de vida. O estudo foi realizado em uma unidade obstétrica do estado do Rio Grande do Sul, Brasil, uma instituição 100\% pública que atende usuárias do Sistema Único de Saúde (SUS) com média de 180 partos mensais. Essa Instituição está passando por mudanças históricas relacionadas às orientações do Ministério da Saúde (MS) quanto à inserção da enfermeira obstétrica na atenção ao parto e nascimento.

Para a análise dos dados, realizou-se estatística descritiva, com frequência absoluta e relativa. Os resultados foram discutidos, observando-se as recomendações do Ministério da Saúde como medidas de boas práticas de atendimento ao parto e nascimento. No que se refere aos aspectos éticos, os dados estão disponíveis, no sistema de registro online da instituição pesquisada, podendo ser usados como fonte de pesquisa.

\section{Resultados}

No que se refere a frequência absoluta e relativa dos indicadores de boas práticas foi evidenciando através do levantamento de dados históricos do hospital um índice de 39,5\% de cesáreas, e 60,5 de partos naturais, sendo 371 cesáreas e 627 partos normais.

As boas práticas recomendadas no manejo do trabalho de parto natural no que se refere a presença de acompanhante, manteve-se ao longo dos meses superior ao parto cesáreo. Porém, pode-se observar um declínio significativo também nas boas práticas com relação ao acompanhamento do familiar no parto natural no período em questão, pois em abril o percentual de acompanhantes foi 98,98 e setembro de 88,3 . O uso de medidas terapêuticas para alivio da dor no trabalho de parto corresponde a $100 \%$ dos atendimentos. A aplicação de pelo menos uma das técnicas não farmacológicas para alivio da dor mantiveram uma porcentagem de $100 \%$ independente da via de parto da parturiente. Entre as medidas ofertadas as parturientes estão: uso da bola, 
massagens, musicoterapia, deambulação e banho. Pode-se observar que essas medidas são ofertadas pela equipe de enfermagem, não sendo uma prática da categoria médica.

Observa-se que embora a dieta esteja sendo ofertada as parturientes em sala de parto independente da forma como ocorreu o parto, ouve um declínio nessa boa prática, atingindo no mês de abril 90, 41\% e em setembro 63,4\%.

As práticas de intervenções de rotina não recomendadas durante o parto natural, como a posição de litotomia, tiveram uma diminuição significativa nos meses de estudo, com uma taxa em abril de 87,76\% e em setembro de $100 \%$ de não utilização. Cabe ressaltar que os dados referentes a posição não litotômica nos partos realizados por Enfermeiras Obstétricas (EOs) foi de $100 \%$ em todos os períodos levantados, predominando o parto de posição de cócoras na banqueta ou cadeira de parto.

A realização de episiotomia ainda apresenta índices considerados significativos na instituição, demonstrando um crescimento ao longo do período estudado. Em abril a taxa de episiotomia foi de 7,14\%, crescendo ao longo dos meses atingindo $12,77 \%$ no mês de setembro. Detectou-se que a prática de episiotomia se restringe ao profissional médico, tendo uma ocorrência de $100 \%$ sem episiotomia dos partos conduzidos pelas EOs.

No que diz respeito a prática de utilização de punção venosa periférica nas parturientes pode-se observar que essa prática é recorrente e de aumento significativo, pois em abril o índice foi de 36,99\% aumentando para 71,24\% em setembro. Observase nos dados evidenciados, que a punção venosa normalmente está associada ao uso de ocitocina sintética, a qual é uma medicação que deve ser usada com precaução, em acordo com as recomendações do MS.

Tabela 1 - Frequência absoluta e relativa dos indicadores de boas práticas.

\begin{tabular}{|c|c|c|c|c|c|c|}
\hline & Abril n(\%) & Maio $n(\%)$ & Junho n(\%) & Julho n(\%) & $\operatorname{Agosto} n(\%)$ & $\begin{array}{l}\text { Setembro } \\
\text { n(\%) }\end{array}$ \\
\hline Parto natural & $98(64,05)$ & $97(61,39)$ & $147(61,49)$ & $96(8,18)$ & $94(56,96)$ & $95(61,44)$ \\
\hline Parto Cesárea & $55(35,95)$ & $60(38,61)$ & $57(38,51)$ & $69(41,82)$ & $71(43,04)$ & $59(38,56)$ \\
\hline $\begin{array}{l}\text { Acompanhante em sala de parto na } \\
\text { cesárea }\end{array}$ & $53(96,36)$ & $58(96,72)$ & $6(98,25)$ & $65(94,2)$ & $68(95,77)$ & $51(86,44)$ \\
\hline $\begin{array}{l}\text { Acompanhamento na sala de parto } \\
\text { natural }\end{array}$ & $97(98,98)$ & $94(96,91)$ & $87(96,7)$ & $97(95,88)$ & $95(96,84)$ & $83(88,3)$ \\
\hline $\begin{array}{l}\text { Alimentação a parturiente } \\
\text { independente da via do parto }\end{array}$ & $66(90,41)$ & $136(86,76)$ & $58(81,6)$ & $72(87,5)$ & $89(74,16)$ & $97(63,4)$ \\
\hline $\begin{array}{l}\text { Posição não litotômica no parto } \\
\text { natural }\end{array}$ & $86(87,76)$ & $91(93,81)$ & $83(92,31)$ & $97(88,66)$ & $86(90,53)$ & $93(100)$ \\
\hline Realização de episiotomia & $7(7,14)$ & $12(12,37)$ & $6(6,59)$ & $8(8,25)$ & $9(9,47)$ & $12(12,77)$ \\
\hline $\begin{array}{l}\text { Realização de punção venosa } \\
\text { independente da via do parto }\end{array}$ & $56(36,99)$ & $88(55,88)$ & $33(46,48)$ & $72(43,06)$ & $48(53,93)$ & $109(71,24)$ \\
\hline
\end{tabular}

Fonte: Banco de dados do sistema de registro local de um Hospital de Ensino no Município Rio Grande, RS, Brasil (2019).

O percentual de contato pele a pele entre mãe e recém-nascido, tanto no parto normal, quanto no parto cesárea mostrara um declínio significativo, variando principalmente no parto cesárea ao apresentar um índice de 26,8\% em abril e 3,57\% em setembro. Da mesma forma, os indicadores evidenciaram que 100\% do contato pele a pele entre mãe e recém-nascido relacionamse aos partos naturais assistidos pelas EOs (Figura 1). 
Figura 1 - Frequência relativa dos indicadores de contato pele a pele entre mãe e recém-nascido de parto natural e cesárea.

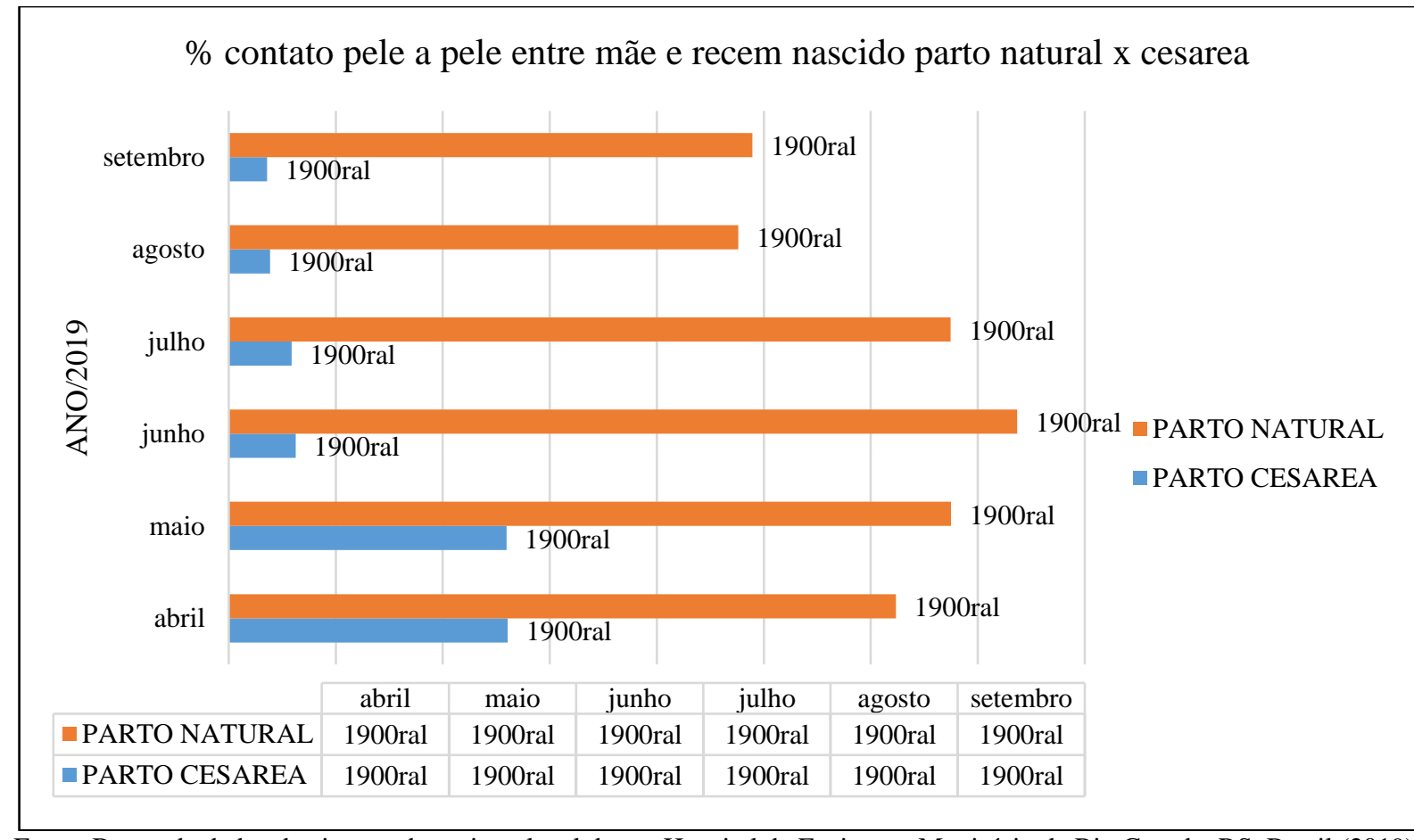

Fonte: Banco de dados do sistema de registro local de um Hospital de Ensino no Município de Rio Grande, RS, Brasil (2019).

O percentual de amamentação na primeira hora de vida nos partos normais foi superior aos partos de cesárea, sendo que os índices sofreram um declínio no período estudado. A variação do índice na cesárea foi de 75\% em abril e 32,14\% em setembro. No parto natural os índices foram $88,8 \%$ em abril e 74,47\% em setembro. No entanto, observou-se que os indicadores de partos normais assistidos por EOs atingiram 100\% de amamentação na primeira hora de vida (Figura 2).

Figura 2 - Frequência relativa dos indicadores de amamentação na $1^{\text {a }}$ hora de vida do recém-nascido de parto natural e cesárea.

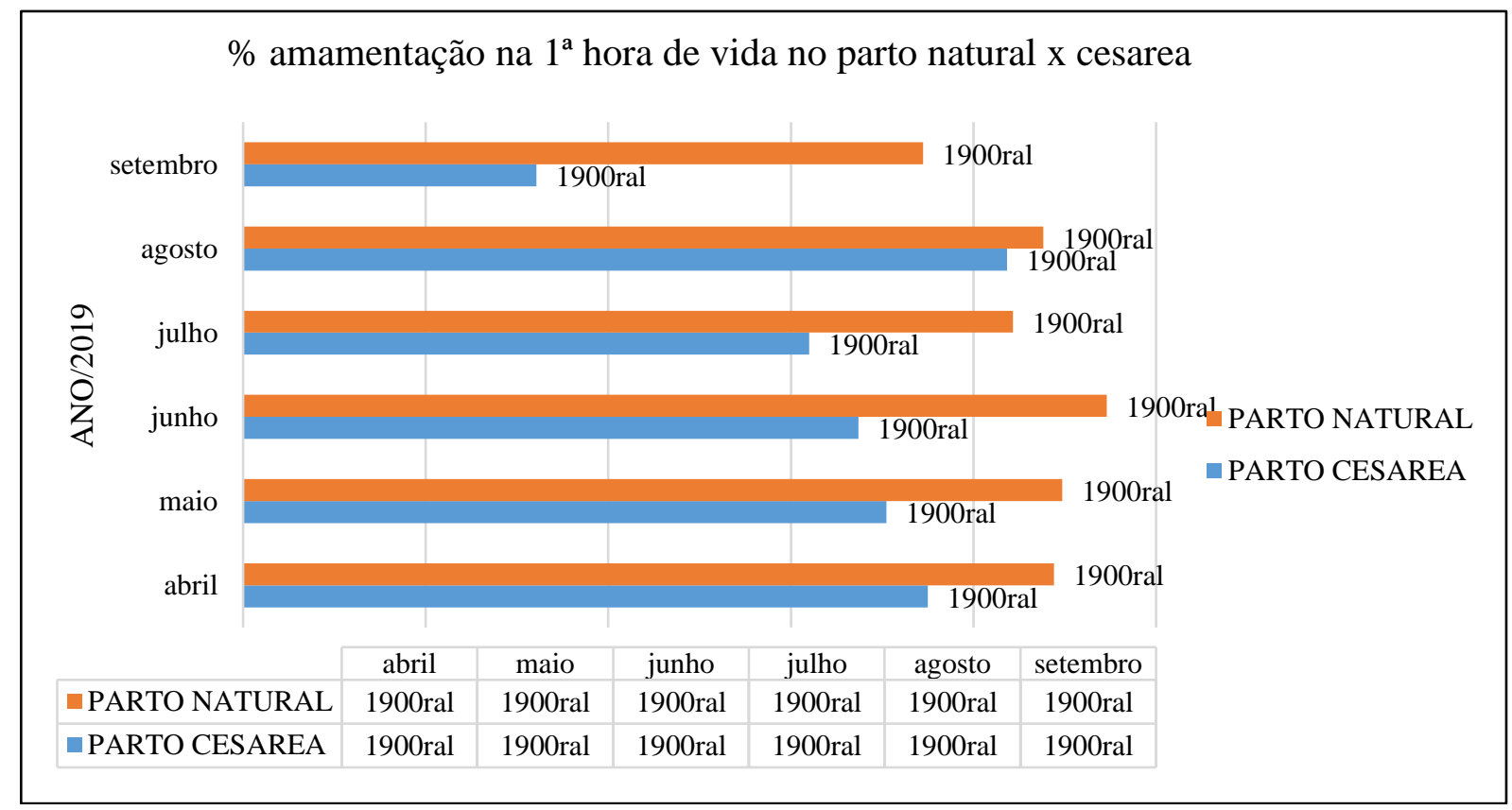

Fonte: Banco de dados do sistema de registro local de um Hospital de Ensino no Município de Rio Grande, RS, Brasil (2019). 
O percentual de clampeamento oportuno do cordão umbilical se apresenta mais elevado nos partos normais, sendo que o clampeamento imediato possui altos índices em ambos os tipos de partos. Aliado a esses índices altos, podemos perceber que os números aumentaram no decorrer do estudo (Figura 3).

Figura 3 - Frequência relativa dos indicadores de clampeamento oportuno do cordão umbilical de parto natural e cesárea.

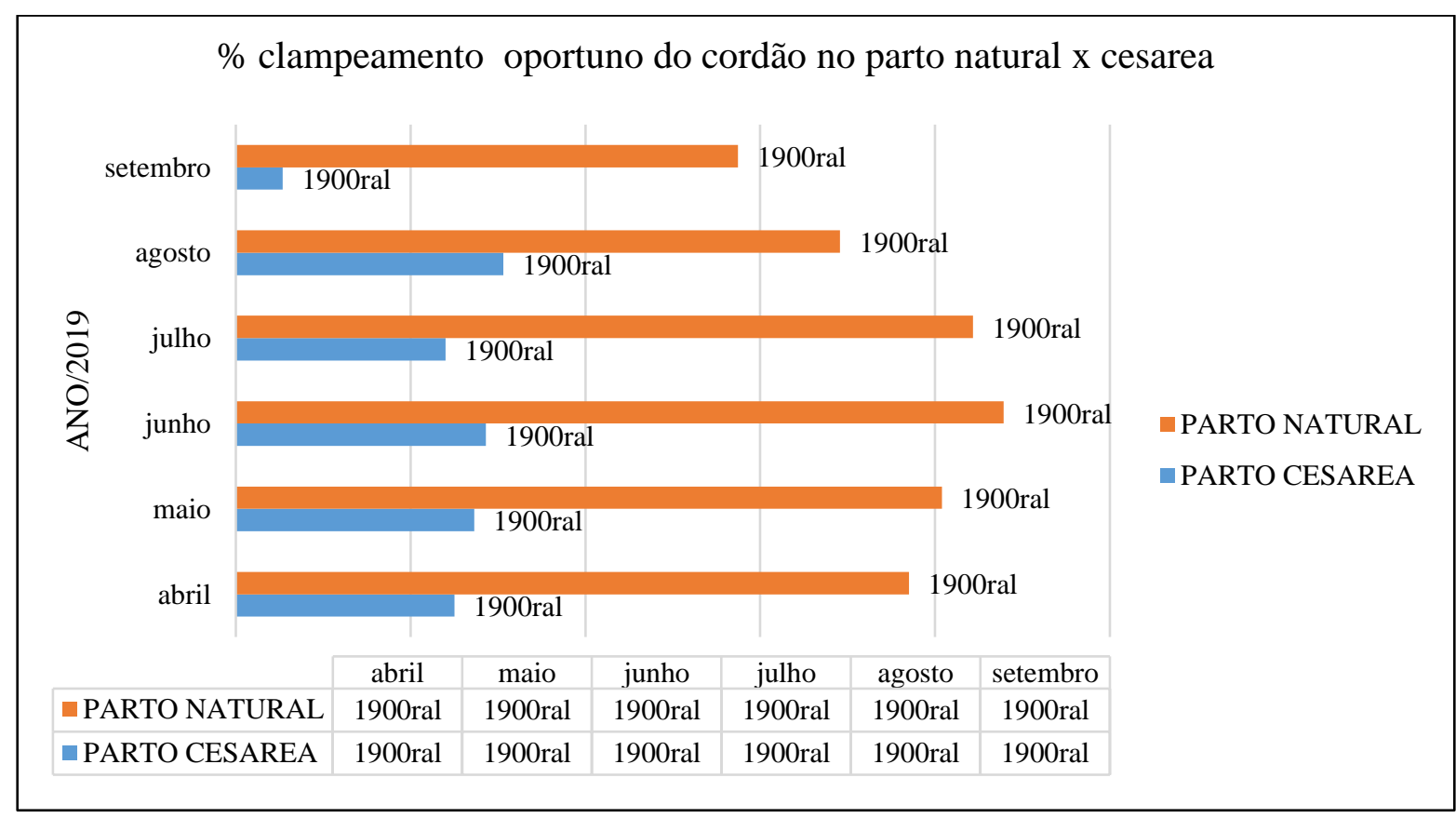

Fonte: Banco de dados do sistema de registro local de um Hospital de Ensino no Município de Rio Grande, RS, Brasil (2019).

Embora os partos assistidos por EOs na instituição estudada seja baixo, variando entre 4\% e 8,79\%, pode-se observar que a assistência prestada por essas profissionais vem ao encontro as medidas adotadas pelo MS como boas práticas de assistência.

\section{Discussão}

O modelo assistencial do hospital de ensino em que foi realizado este estudo aderiu, em 2019, ao Projeto Apice On, sendo este um projeto de aprimoramento e inovação no Cuidado e Ensino em Obstetrícia e Neonatologia, se comprometendo na melhoria do atendimento da gestante no ciclo gravídico puerperal. Entre as medidas acordadas está a inserção da Enfermeira Obstétrica (EO) na assistência ao parto de risco habitual. Essa inserção das EOs encontra-se em processo de implantação com a criação de protocolos para essa assistência. Assim, apesar de estar em processo de implementação, a instituição conta com EOs em todos os turnos de trabalho. Dessa forma, algumas parturientes já estão sendo assistidas por essas, dando início a um cuidado compartilhado com a equipe médica num processo de transição do modelo tradicional predominante até então.

Observa-se que altos índices de cesárias ainda são predominantes na instituição pesquisada variando entre 35,95\% e $42,7 \%$. Não obstante, um estudo realizado mostrara que ouve uma redução de $8 \%$ de parto por cesariana após a inserção das EOs na assistência ao parto de risco habitual (Medeiros, et al., 2016).

Assim, este estudo corrobora com os índices do Brasil, que é considerado um país que presta um atendimento intervencionista, onde a taxa de cesárea expressa esse modelo sendo umas mais elevadas do mundo. Segundo o MS a taxa de operação de cesariana no Brasil situa-se em torno de 56\%, aumentando assim o risco de mortalidade materna, perinatal ou neonatal. Pode-se evidenciar que os índices de cesáreas extrapolam o preconizado pelo MS, mesmo diante da inserção de 
protocolos de boas práticas para a sua diminuição. Nesse sentido, considerando as características da população obstétrica brasileira, a taxa de referência estimada é de aproximadamente 29\% (Brasil, 2015).

Pode-se observar que muitos partos ocorreram sem a presença de acompanhante, tendo inclusive uma redução da presença do mesmo no parto cesáreo de $96,36 \%$ a $86,44 \%$, e no parto normal uma variação de $98,98 \%$ a 88,3 durante o período do estudo. A lei 11.108 de 2005, permite o acompanhamento da mulher no ciclo gravídico puerperal de escolha da mulher grávida, no entanto pode-se observar que apesar da criação da lei há mais de uma década esse direito ainda não é respeitado na sua totalidade (Brasil, 2005). Mafetoni e Shimo (2014), em seu estudo, indica que o acompanhante na sala de parto contribui para o aumento nas taxas de partos normais e redução do tempo de trabalho de parto, preconizando o esperado para um parto mais humanizado.

Observa-se que a utilização de pelo menos um método não farmacológico para alivio da dor ocorreu em 100\% dos atendimentos feitos na instituição. Esses métodos caracterizam-se por terem baixo custo, apresentando grande eficácia no alivio da dor, principalmente quando ofertados de forma associada, e melhora da qualidade da assistência (Medeiros, et al., 2016).

No que se refere ao uso dos métodos não farmacológicos para o alívio da dor, há estudos que relatam que essas práticas influenciam positivamente na fisiologia do parto, promovendo sensação de bem-estar e auxiliando a parturiente na progressão do trabalho de parto. Dentre elas destacam-se: o uso da bola suíça, o banho morno, massagem, deambulação, escalda pés, agachamento e uso da banqueta (Costa et al., 2021; Gupta, et al., 2017; Pereira, et al., 2018; Pereira, et al., 2018).

Pode-se evidenciar que, na instituição pesquisada, além do uso de métodos não farmacológicos para o alívio da dor, existe a prevalência de outras ações consideradas como boas práticas preconizadas pelo MS/OMS, tais como: liberação de dieta líquida para a parturiente, incentivo de posições verticais no segundo período do parto e a presença do acompanhante de escolha da mulher, qualificando o cuidado prestado, reduzindo as intervenções desnecessárias, demonstrando um cuidado humanizado e valorizando o trabalho desenvolvido pelas Enfermeiras Obstétricas (Costa et al., 2021; Oliveira \& Penna, 2017).

Apesar das boas práticas reduzirem as taxas de práticas intervencionistas sem a adequada indicação clínica e embasamento científico, ainda é muito incipiente estas ações no hospital pesquisado. O não uso de algumas boas práticas recomendadas pelo MS, configuram violência obstétrica e nem sempre são reconhecidas pela paciente e familiares. No entanto, sabe-se que os profissionais envolvidos na assistência ao parto conhecem o que é caracterizado como violência obstétrica, bem como, os direitos das parturientes (Amaral, et al., 2019).

Destarte, pode-se observar nesse estudo que a liberação da dieta, teve uma redução nos índices de 90, 41\% para 63,4\%, entretanto, a posição não litotômica teve um acréscimo significativo ao longo do período estudado, sendo de 87,76\% para $100 \%$. Essa conduta, vem ao encontro das boas práticas preconizadas pelo MS. Segundo Cavalcanti, et al. (2019), os benefícios decorrentes das posições não litotômicas devem ser informados, bem como, encorajados as parturientes.

A episiotomia, nesse estudo, teve uma variação ao longo do período estudado, bem como o uso de punção venosa periférica. Essa variação mostra um aumento significativo na pratica de episiotomia de 7,14\% a 12,77\%. Da mesma forma, o uso de punção periférica passou de 36,99\% para 71,24\%. Esse procedimento tem sido depreciado por ser considerado, muitas vezes, inoportuno. Assim sendo, segundo Leal, et al. (2014), essas intervenções ocorrem principalmente em instituições públicas, e com mulheres de menor nível de educação.

Nesse seguimento, a punção venosa, na maior parte dos casos, está associada ao uso de ocitocina sintética para indução, aceleração ou correção de alterações na evolução do trabalho de parto, sendo considerada, na maioria das vezes, uma intervenção desnecessária. Pode-se atribuir o uso indiscriminado da ocitocina ao modelo tecnocrático sobre as práticas dos profissionais, o que reproduz uma assistência intervencionista (Oliveira \& Penna, 2017).

Com relação ao contato pele a pele na primeira hora de vida, iniciado imediatamente ao nascimento entre o binômio mãe-bebê, que se encontra entre os dez passos para o sucesso do aleitamento materno - recomendados pela Iniciativa Hospital 
Amigo da Criança (IHAC), pode-se observar nesse estudo uma oscilação das taxas, chegando ao mínimo de 47,62\% no parto normal e 3,57\% no parto cesárea. O contato pele a pele na primeira hora de vida, juntamente com o alojamento conjunto mãebebê, foram algumas medidas adotadas em países de primeiro mundo afim de aumentar os índices de aleitamento materno (Nascimento, et al., 2019).

Assim, apesar de alguns estudos demonstrarem que o contato pele a pele precoce no binômio mãe - bebê, seja um procedimento sem custos, seguro e com benefícios ao bebê, ele ainda é negligenciado pelos profissionais de saúde. A prática de colocar os recém-nascidos em contato pele a pele na primeira hora de vida reflete a forma de organização da instituição, bem como, as rotinas hospitalares, constituindo-se num dos passos mais fáceis de ser instalado (Sampaio et al., 2016).

Reforçando essa prática, o MS preconiza que os recém-nascidos, sem intercorrências no nascimento, devem ser inicialmente avaliados, sendo a realização dos primeiros cuidados como a promoção de calor e a liberação das vias aéreas, realizados junto à mãe, oportunizando o contato pele a pele.

Outra indicação que não é preconizada pelo MS é o clampeamento precoce, isto é, aquele realizado imediatamente após o nascimento. O MS considera não existir uma indicação científica para o clampeamento precoce, destacando que o clampeamento tardio, considerado após um, dois ou três minutos que cessarem as pulsações, previne a anemia no primeiro ano de vida do bebê (Oliveira \& Penna, 2017; Sampaio, Bousquat \& Barros, 2016).

No entanto, apesar das evidências vantajosas do clampeamento tardio, observa-se que na instituição pesquisada, existe um aumento significativo do procedimento precoce nas cesáreas, aumentando de $25 \%$ no início do estudo para 74,47\% no final do estudo. Tal como, o clampeamento de cordão tardio no parto normal, apresentara um decréscimo de 77,01\% para 74,47\%.

Atribui-se o clampeamento precoce a liberação do binômio mãe-bebê mais rápido da sala de parto e a ansiedade do pediatra em dar assistência ao recém-nascido. Essa atividade, na maioria das vezes, evidencia a priorização dos cuidados centrados na técnica e, dessa forma, menospreza o cuidado holístico e humanizado (Sampaio et al., 2016).

Estudos recentes trazem uma incidência elevada da prática de episiotomia realizada pelo profissional médico (Medeiros, et al., 2016). Tais trabalhos, corroboram com os resultados da presente análise, que apresentou uma variação de 4,21\% a 12,37\% de episiotomias realizadas por estes profissionais. Em contrapartida, $100 \%$ das mulheres assistidas por EOs, na instituição investigada, não foram submetidas a episiotomia.

Em consonância com esses achados, Medeiros, et al. (2016) afirma que a inserção da EO tem modificado o modelo intervencionista no parto de risco habitual, sugerindo que as EOs vêm com um novo perfil assistencial. As EOS utilizam uma prática menos intervencionista na fisiologia do parto, tais como as medidas não farmacológicas para o alívio da dor e o respeito a posição de escolha das parturientes; para mais, prestam um cuidado humanizado e de qualidade, estando suas práticas em consonância com o que é preconizado pelo MS/OMS.

A assistência médica encontrada mostra-se ainda incipiente, pois muitas de suas ações precisam ser modificadas, tendo em vista a busca do MS por uma assistência integral e humanizada. Desse modo, o estudo evidencia que o cuidado da EO tem se mostrado alinhado aos preceitos da humanização da assistência ao parto e ao nascimento, ao passo que a inserção dessas profissionais no contexto estudado reconfigurou atitudes e práticas em saúde implementadas pelos agentes atuantes no campo obstétrico e neonatal (Medeiros, et al., 2016). Tais achados, corroboram com os resultados da presente investigação, que demonstra que as boas práticas no trabalho de parto de risco habitual assistidos por EOs, a partir do respeito a escolha da posição para parir, da implementação do contato pele a pele do binômio mãe-bebê na primeira hora após o parto, do auxílio a prática da amamentação, entre outras, obtiveram $100 \%$ de êxito.

Dessa forma, estudos nacionais e internacionais atribuem a inserção das EOs ao modelo de assistência preconizado pelo MS, pois as EOs buscam enfocar aspectos fisiológicos e emocionais da parturição, contribuindo para a diminuição de índices de 
intervenções desnecessárias no processo fisiológico do parto, passo fundamental na assistência ao parto humanizado e para maior satisfação das pacientes ao atendimento ofertado (Beque, et al., 2020; Medeiros, et al., 2016; Oliveira, et al., 2021; Vitral, 2017).

\section{Conclusão}

Os resultados dos estudos dos indicadores da assistência obstétrica, realçam um aumento significativo do acesso as informações apropriadas ao parto e nascimento, demonstrando um aumento da proporção de uso de práticas benéficas e a redução de práticas consideradas prejudiciais. Constatou-se que ainda há um predomínio de altas taxas de cesariana, sendo essas, explicadas através da Classificação de Robson, pois a instituição estudada é referência em atendimento a gestante de alto risco. Apesar dos indicadores demonstrarem uma modificação do perfil de atendimento a parturiente, com a ocorrência do aumento de boas práticas preconizadas pelo MS, observa-se ainda uma assistência ao parto pautada em medicações e no uso cauteloso de técnicas e intervenções preconizadas.

Ademais, evidenciou-se no estudo, que, apesar das tentativas institucionais de aplicar boas práticas do MS na assistência obstétrica, as práticas medicamentosas, bem como, a instituição de intervenções desnecessárias e prejudiciais, como a realização da episiotomia, ainda se encontram presentes na instituição pesquisada. Essas práticas, distanciam-se das recomendações do MS no que se refere ao atendimento humanizado e ao respeito aos direitos das mulheres. Por fim, a inserção de EOs na assistência dos partos de risco habitual se faz necessária, a fim de contribuir na melhoria do serviço desenvolvido pela instituição e na busca da autonomia das parturientes.

Assim, percebe-se que existe um grande desafio na busca pela transformação da assistência às mulheres e aos recémnascidos, em direção as boas práticas propostas pelo Ministério da Saúde e pelo Programa Apice On. Essas modificações, dependem diretamente do conhecimento dos gestores em saúde acerca das boas práticas propostas, da incorporação de capacitações dos profissionais atuantes nos Centros Obstétricos, do aprimoramento estrutural dos serviços, bem como, do enfrentamento das distintas classes profissionais da área, que em conjunto, desempenham o atendimento a parturiente. Posto isto, busca-se a partir das considerações deste estudo disseminar produções científicas e avanços existentes na área de modo a contribuir com a diminuição das práticas prejudiciais que ainda se perpetuam e são instituídas às mulheres.

Por fim, sugere-se a expansão do presente estudo para outras regiões do país para que seja possível identificar não somente a realidade de uma única instituição de saúde, bem como, recomenda-se a realização de pesquisas que evidenciem a implementação e a atuação das EOs nos Centros Obstétricos.

\section{Referências}

Amaral, R. D. C. S. A. et al (2019). The insertion of the nurse midwife in delivery and birth: obstacles in a teaching hospital in the Rio de Janeiro state. Escola Anna Nery, 23 (1). http://dx.doi.org/10.1590/2177-9465-ean-2018-0218

Brasil. (2005). Lei 11.108, de 7 de abril de 2005. Dispõe sobre o direito de acompanhante durante o trabalho de parto, parto e pós-parto imediato. Diário Oficial da República Federativa do Brasil. http://www.planalto.gov.br/ccivil_03/_Ato2004-2006/2005/Lei/L11108.htm

Brasil. (2017). Ministério da Saúde. Diretrizes nacionais do parto humanizado. https://portaldeboaspraticas.iff.fiocruz.br/wp-content/uploads/2018/07/dire trizesnacionaisassistenciapartonormal.pdf

Brasil. (2011). Ministério da Saúde. Secretaria de Assistência à Saúde. Departamento de Ações Programáticas Estratégicas. Institui, no âmbito do Sistema Único de Saúde (SUS) a Rede Cegonha. https://www.saude.gov.br/saude-para-voce/saude-da-mulher/rede-cegonha

Brasil. (2015). Ministério da Saúde. Secretaria de Assistência à Saúde. Redefine as diretrizes para implantação e habilitação de Centro de Parto Normal (CPN), no âmbito do Sistema Único de Saúde (SUS), para o atendimento à mulher e ao recém-nascido no momento do parto e do nascimento, em conformidade com o componente Parto e Nascimento da Rede Cegonha, e dispõe sobre os respectivos incentivos financeiros de investimento, custeio e custeio mensal. https://bvsms.saude.gov.br/bvs/saudelegis/gm/2015/prt001107012015.html

Brasil. (2020). Ministério da Saúde. Secretaria de Vigilância em Saúde. Boletim Epidemiológico N $^{\circ} 20$ - maio 2020.51 (20). https://portaldeboas praticas.iff.fiocruz.br/biblioteca/boletim-epidemiologico-no-20-maio-2020/

Beque, J. T. S. et al (2020). Gerenciamento da enfermeira obstétrica nas boas práticas no parto e nascimento. Research, Society and Development, 9 (11), 
Research, Society and Development, v. 11, n. 2, e2611225319, 2022

(CC BY 4.0) | ISSN 2525-3409 | DOI: http://dx.doi.org/10.33448/rsd-v11i2.25319

e939119526-e939119526. http://dx.doi.org/10.33448/rsd-v9i11.9526

Cavalcanti, A. C. V. et al (2019). Terapias complementares no trabalho de parto: ensaio clínico randomizado. Revista Gaúcha de Enfermagem, 40 , e20190026. https://doi.org/10.1590/1983-1447.2019.20190026

Costa, R. de S., Ferreira, J. P., \& Viana, M. R. P. (2021). Boas práticas na assistência ao parto natural. Research, Society and Development, 10 (5), e53210515394e53210515394. http://dx.doi.org/10.33448/rsd-v10i5.15394

Gupta, J. K. et al (2017). Position in the second stage of labour for women without epidural anaesthesia. Cochrane database of systematic reviews, (5). 10.1002/14651858.CD002006.pub4.

Leal, M. D. C. et al (2014). Intervenções obstétricas durante o trabalho de parto e parto em mulheres brasileiras de risco habitual. Cadernos de Saúde Pública, 30, S17-S32. https://doi.org/10.1590/0102-311X00151513

Mafetoni, R. R., \& Shimo, A. K. K. (2014). Non-pharmacological methods for pain relief during labor: integrative review. Rev Min Enferm, 18(2), 513-20. $10.33448 /$ rsd-v10i7.16446.

Medeiros, R. M. K. et al (2016). Humanized Care: insertion of obstetric nurses in a teaching hospital. Revista brasileira de enfermagem,69, e1091-1098. https://doi.org/10.1590/0034-7167-2016-0295

Nascimento, S. L. D. et al (2019). Conhecimentos e experiências de violência obstétrica em mulheres que vivenciaram a experiência do parto. Enfermería Actual de Costa Rica, (37), 66-79. http://dx.doi.org/10.15517/revenf.v0ino.37.35264

Oliveira, L. L. F. D. et al (2021). Caracterización de la atención obstétrica desarrollada en la enseñanza de los hospitales de una capital del noreste de Brasil. Revista Brasileira de Enfermagem, 75, e20200896. https://doi.org/10.1590/0034-7167-2020-0896

Oliveira, V. J., \& Penna, C. M. D. M. (2017). Discussing obstetric violence through the voices of women and health professionals. Texto \& ContextoEnfermagem, 26. https://doi.org/10.1590/0104-07072017006500015

Pereira, P. S. L. et al (2018). Tecnologias não invasivas de cuidado: percepção das puérperas. Rev. enferm. UFPE on line, 2129-2136. https://doi.org/10.5205/1981-8963-v12i8a236584p2129-2136-2018

Pereira, S. B. et al (2018). Boas práticas de atenção ao parto e ao nascimento na perspectiva de profissionais de saúde. Revista Brasileira de Enfermagem, 71 , 1313-1319. https://doi.org/10.1590/0034-7167-2016-0661

Sampaio, Á. R. R., Bousquat, A., \& Barros, C. (2016). Contato pele a pele ao nascer: um desafio para a promoção do aleitamento materno em maternidade pública no Nordeste brasileiro com o título de Hospital Amigo da Criança. Epidemiologia e Serviços de Saúde, 25, 281-290. https://doi.org/10.5123/S167949742016000200007

Vitral, G. L. (2017). Clampeamento oportuno de cordão umbilical e suas repercussões na concentração de hemoglobina neonatal. SaBios-Revista de Saúde e Biologia, 12(1), 35-41. Recuperado de https://revista2.grupointegrado.br/revista/index.php/sabios/article/view/2062

Vogt, S. E., Silva, K. S. D., \& Dias, M. A. B. (2014). Comparação de modelos de assistência ao parto em hospitais públicos. Revista de Saúde Pública, 48, 304313. https://doi.org/10.1590/S0034-8910.2014048004633 